\title{
THE EFFECTS OF REPRESENTATION MODE ON CONCEPTUAL COHERENCE IN THE DESIGN OF PHYSICAL PRODUCTS
}

\author{
Trinh, Yen Mai Thi; Elverum, Christer Westum \\ Norwegian University of Science and Technology
}

\begin{abstract}
Coherence is important in the design of products, because it makes them easier to understand for their users. Designers can use different representation modes to express ideas about coherence. However, perception of a representation can be influenced by its mode. Therefore, designers must be aware of the influences that different representation modes can have on perception of coherence.

The aim of the present study was to investigate the effects of experienced modality on perception of conceptual coherence. In the study, participants were divided into two conditions for two representation modes: written word and physical object, both representing the same concepts. Each participant was presented with the concepts as words or objects and asked to list the properties of each concept. The results showed that between the two conditions, frequencies of responses were similar while frequencies of response contents were dissimilar and sometimes contrary. The main findings suggest that the effects of the modalities, written word and physical object, do not differ significantly for mental activity, but do differ considerably for semantic processing in ways that has implications for design.
\end{abstract}

Keywords: Design engineering, Design practice, Design methods, Design representation

\section{Contact:}

Trinh, Yen Mai Thi

Norwegian University of Science and Technology

Department of Mechanical and Industrial Engineering

Norway

yen.m.t.trinh@ntnu.no

Cite this article: Trinh, Y.M.T., Elverum, C.W. (2019) 'The Effects of Representation Mode on Conceptual Coherence in the Design of Physical Products', in Proceedings of the 22nd International Conference on Engineering Design (ICED19), Delft, The Netherlands, 5-8 August 2019. DOI:10.1017/dsi.2019.172 


\section{INTRODUCTION}

Objects should enhance people's well-being rather than complicate their lives. Designers should therefore strive to design products that require the minimum amount of mental effort for users to understand. To limit the mental effort needed to understand things, people establish mental models from experiences with objects in the world (Athavankar, 1989). Mental models of objects embody beliefs about conceptual coherence (Medin, 1989) and can be referred to for explanations about other objects (Norman, 2013). Hence, designers must ensure conceptual coherence in the design of products that are to be effortlessly understood by their users.

As mental models reside in the minds of people, designers can use external representation to explore internal thoughts pertinent to conceptual coherence (Goldschmidt and Porter, 2004). However, since mode of representation influences perception (Schifferstein and Hekkert, 2008), different representation modes of the same concept can be perceived differently. In the development of physical products, it is common to work with many different representation modes, such as notes, sketches and tangible models. Thus, it is important to be aware of how different representation modes influence the perception of conceptual coherence. In this paper, we present a study that sets out to investigate how conceptual coherence is perceived for a written word representation in comparison to a physical object representation of concepts.

\section{BACKGROUND}

\subsection{Change in product development}

Changes that occur in the life cycle of a product often lead to process or product changes, which can cause increased resource cost, decreased product relevance, and compromised innovativeness (Ullah et al., 2016; Smith, 2007; Kidd and Thompson, 2000; Thomke and Reinertsen, 1998).

Technological advances have created more possibilities to manipulate separate product properties without changing others (Schifferstein and Desmet, 2008), which can accommodate change by facilitating flexibility in the product development process (Smith, 2007). For the development of physical products, modularisation of architecture is one approach to facilitate flexibility. A modular architecture allows changing chunks independent of each other, as it is considered in terms of its functional physical chunks and the interactions between them to provide the overall intended main function (Ulrich and Eppinger, 2012; Smith, 2007; Gershenson et al., 2003). This implies a distinction between function and form, which is not uncommon in product development (Townsend et al., 2011; Schifferstein and Hekkert, 2008; Chitturi et al., 2007; Smith, 2007). However, a product for which there is distinction between its function and form, and further distinction between its functional physical chunks, carries the inherent danger of lacking coherence (Schifferstein and Desmet, 2008). A product in which its properties cohere with each other provides a coherent message that clarifies what it is, what it does, and how it can be operated, and thus makes the product understandable.

\subsection{Coherence in product design}

The term coherence generally describes the quality of forming a unified whole (Oxford Dictionaries, 2018). In product design, coherence usually refers to relationships internal in and external to a product. External coherence can be considered between a product and its brand identity when the product is 'evoking certain associations that . . . are aligned to strategically defined message of the brand' (Karjalainen, 2003); between a product and its line members when a group of same brand products 'share similar features, functionality, or lineage' (Bowman, 2006) or are 'sold to the same customer groups' (Kotler and Armstrong, 2012); and between a product and its context when there is 'fit as a whole to the context outside' (Alexander, 1973). Internal coherence can be considered between a product's properties when there is 'internal fitness between the pieces it is made of' (Alexander, 1973); between the product's form and function when they are 'mutually united' (Su and Hao, 2010); and between the product's sensory information when there is 'cross-modal correspondence' or 'congruence of sensory messages' (Schifferstein and Hekkert, 2008) or when 'sensory information duplicate or complement one another' (Schifferstein and Desmet, 2008). Coherence is thus illustrated as a relationship between properties in an entity or between the entity and other context entities based on "hanging-togetherness". 


\subsection{Coherence is perceptual}

The notion of "hanging-togetherness" corresponds to theories about conceptualisation and categorisation from cognitive psychology research (Murphy and Medin, 1985; Smith and Medin, 1981), which is concerned with understanding the thought processes behind sense-making and decision-making of people while interacting with natural and artificial objects (e.g., Murphy, 1991; Rosch et al., 1976). The theories suggest that people have conceptual and categorical beliefs about the objects that they experience, which they refer to when they encounter other objects (Norman, 2013; Athavankar, 1989). Through experiences with real objects in everyday life, a person will establish and hold some presupposed beliefs about properties that do and do not co-occur (Anderson, 2015; Schifferstein and Hekkert, 2008). The person will build mental models of concepts upon existing beliefs as well as inferences from information identified in the product itself (Norman, 2013; Burnston and Cohen, 2012). Mental models are internal thoughts that influence the construction of explanations, the interpretation of information, the revision of theory (Vosniadou, 2002), and that embody beliefs about conceptual coherence (Medin, 1989). Most people within a culture tend to share mental models about the synthesis of properties that are perceived as being coherent for a particular concept with concepts of categories that are common to their culture (Rosch et al., 1976). However, for the same concept, it is possible for one person to have one or several different mental models (Norman, 2013), as is it possible for different people to have different or overlapping ones.

\subsection{Design is representation}

What the designer intended a product to be and what the user perceives the product to be can be different (Crilly et al., 2008). Designers can use different modalities to represent one concept, but users may perceive each modality differently. As representations of a concept, the label of the concept, the image of the concept, and the true concept itself are different modalities. For example, the uttered word 'moon' is different from the written word moon, which is different from the image $\mathbb{C}$, which is different from the observable physical entity that orbits the Earth known as the Moon.

For designers, designing cannot be performed without representation (Goldschmidt and Porter, 2004). External representations, such as spoken utterances, written notes, two-dimensional sketches, or threedimensional models, can be used to express anything from abstract to concrete ideas. In particular, external representations can be used to reflect internal thought processes to be read or transformed (Brereton, 2004), and can therefore be used to explore perception of conceptual coherence. However, perception of a representation greatly depends on the mode of the information that it provides (Schifferstein and Hekkert, 2008), so designers must be aware of how different representation modes influence the perception of conceptual coherence.

Despite the interest in effects of different modalities on cognition (e.g., Dilkina and Lambon Ralph, 2012; Ginns, 2005), the area of modality effects on cognition with respect to design needs extended research (Athavankar, 1989). Thus, the goal of this research is to identify the effects of different representation modes on cognition and their implications for design by first addressing the question: What are the differences in effects on cognitive processing of conceptual coherence between a written word representation and a physical object representation?

\section{STUDY}

The qualitative method of feature listing was adopted for the present study. A mental model of a concept comprises syntheses of properties that are perceived as being coherent for the concept, so properties that are true of the concept are associated with it. In two conditions for two modalities each representing the six same concepts, participants' perception of conceptual coherence for the concepts were obtained by asking them to list the properties of the concepts.

Assuming that people of the same culture will associate the same properties with the same concept that is common to their culture, it was expected that a written word representation and a physical object representation for the same concept would call to mind the same properties, and thus elicit responses of the same semantic content. Additionally, considering that physical objects are richer in sensory information, it was also expected that a physical object would call to mind a greater number of properties, and hence elicit higher quantity of responses. 


\subsection{Method}

\subsubsection{Participants}

Participants were 44 academic individuals, including 28 males and 16 females between the age of 21 to 36 years. Perceived associations between concepts and properties can be influenced by culture (Unsworth et al., 2005), expertise (Tanaka and Taylor, 1991), and experience (Murphy and Medin, 1985). As control for these factors, only participants residing in Norway and of engineering branches were included. Participants were randomly allocated to either of two conditions, word condition or object condition, and were evenly divided between them.

\subsubsection{Stimuli}

The stimuli were two modalities: written word and physical object representing six concepts belonging to three categories of artificial concrete objects common to the Scandinavian culture: furniture, clothing, and tableware. Concepts were intended to be presented at a basic level, where they represent typical instances that contain properties most representative of the concepts (Rosch et al., 1976), and thus are most salient. However, pilot studies revealed that only the written word representations were perceived at a basic level while the physical object representations were perceived at a subordinate level. Hence, for fairer comparability between the two modalities in the present study, the concepts were presented at a subordinate level. From each of the three categories, two concepts were chosen; folding chair and table lamp from the furniture category, T-shirt and ankle sock from the clothing category, and dessert plate and water glass from the tableware category. Participants were exposed to the written word representation of each concept in the word condition and to the physical object representation of each concept in the object condition.

\subsubsection{Procedure}

Participants were asked to complete a feature listing task. They were individually presented with either the written word representation or the physical object representation of the concepts in the order: folding chair, T-shirt, dessert plate, table lamp, ankle sock, and water glass. They were asked to respond to each representation by listing the properties that they considered classified the representation as its concept. Following the presentation of each concept, the participant was listing its properties continuously for 2 minutes, after which they were stopped and the representation was removed and replaced with another representation. This process was repeated until the participant had responded to the representations of all concepts in the same mode.

\subsubsection{Content analysis}

All raw data was accumulated into one comprehensive data set. The data was first organised into single items of semantically individual responses. While still distinguished as responses from the word condition and from the object condition, the items were then grouped according to their semantic content. Frequencies of items as individual properties were counted.

Following the data content, items were categorised into three property types that each in an object can be experienced in a similar nature: physical characteristics, pragmatic abilities, and hedonic qualities. The physical characteristics category included items that referred to properties describing either weight (e.g., light, heavy), size (e.g., small, big), shape (e.g., cylindrical, boxy), colour (e.g., white, red), pattern (e.g., stripes, check), texture (e.g., smooth, rough), material (e.g., cotton, metal), durability (e.g., fragile, stable), or part (e.g., seat, handle). The pragmatic abilities category included items that referred to properties describing either utility (e.g., wearable, cooling), usability (e.g., usable, suitable), or use context (e.g., used at the hospital, used during winter). Here, while the content of use context indicates that it belongs to the pragmatic abilities category, they were not considered in the final data set, since the study was interested in investigating perception of conceptual coherence independent of elements external to a concept. The hedonic qualities category included items that referred to properties describing emotion (e.g., comfortable, boring) or aesthetic (e.g., ugly, plain). All items that were not denoting a property (i.e., attribute, feature, characteristic, element, quality, or ability) were disregarded and not considered in the final data set. Frequencies of items as property types were counted. 


\subsection{Results}

From a comparison of the frequencies of individual properties between the word condition and the object condition, no complete agreement was observed (Table 1. Data excerpt; complete data set is available from the authors upon request). For some concepts, some properties that occurred relatively often within one condition, occurred relatively often within the other condition. For example, for the concept table lamp, the property it can emit light occurred more often than other properties in both conditions. Contrarily, for all concepts, some properties that occurred relatively often within one condition, occurred relatively rarely within the other condition. For example, also for the concept table lamp, the property it is electrical occurred often in the object condition, but occurred rarely in the word condition. Additionally, for some concepts, some properties that occurred in the word condition had conflicting semantic content to some properties that occurred in the object condition, of which some properties were directly observable in the object condition. For example, for the concept $T$-shirt, the property white occurred in the word condition, while the property black occurred in the object condition, where the T-shirt presented in the object condition was indeed black.

Table 1. Frequencies of properties of concepts for word condition and object condition

\begin{tabular}{|c|c|c|c|}
\hline & & Word condition & Object condition \\
\hline Concept & Property & Occurences & Occurences \\
\hline \multirow[t]{4}{*}{ Folding chair } & You can sit on it & 9 & 14 \\
\hline & It is of wood & 3 & 13 \\
\hline & You can fold it & 5 & 8 \\
\hline & It has legs & 1 & 10 \\
\hline \multirow[t]{4}{*}{ T-shirt } & It has short sleeves & 9 & 6 \\
\hline & It is of cotton & 6 & 8 \\
\hline & You can wear it & 4 & 7 \\
\hline & It is soft & 2 & 9 \\
\hline \multirow[t]{4}{*}{ Dessert plate } & It is round & 4 & 14 \\
\hline & It is ceramic & 2 & 13 \\
\hline & It is small & 11 & 2 \\
\hline & It is flat & 1 & 10 \\
\hline \multirow[t]{4}{*}{ Table lamp } & It can emit light & 25 & 17 \\
\hline & It has a lamp shade & 4 & 15 \\
\hline & It is electrical & 2 & 16 \\
\hline & It has a light bulb & 7 & 7 \\
\hline \multirow[t]{4}{*}{ Ankle sock } & It can cover a foot & 8 & 8 \\
\hline & It is warming & 6 & 7 \\
\hline & It is of cotton & 5 & 8 \\
\hline & It is white & 8 & 2 \\
\hline \multirow[t]{4}{*}{ Water glass } & It is transparent & 13 & 16 \\
\hline & It is of glass & 12 & 7 \\
\hline & It is cylindrical & 5 & 13 \\
\hline & In can contain liquid & 4 & 12 \\
\hline
\end{tabular}

A comparison of frequencies of property types between the two conditions showed some agreement. Across both conditions, the individual properties that occurred most often were properties describing physical characteristics (Figure 1). In the word condition and object condition, the properties describing physical characteristics occurred most often with $46 \%$ and $61 \%$ respectively, properties describing pragmatic abilities occurred less often with $43 \%$ and $33 \%$ respectively, and properties describing hedonic qualities occurred least often with $11 \%$ and $6 \%$ respectively. The frequencies of property types were not equal between the two conditions, but the distributions of property types were similar across the two conditions. The property types that occurred relatively often within one 
condition, occurred relatively often within the other condition. However, the distribution of property types describing physical characteristics and pragmatic abilities varied more across concepts in the word condition than in the object condition.

a

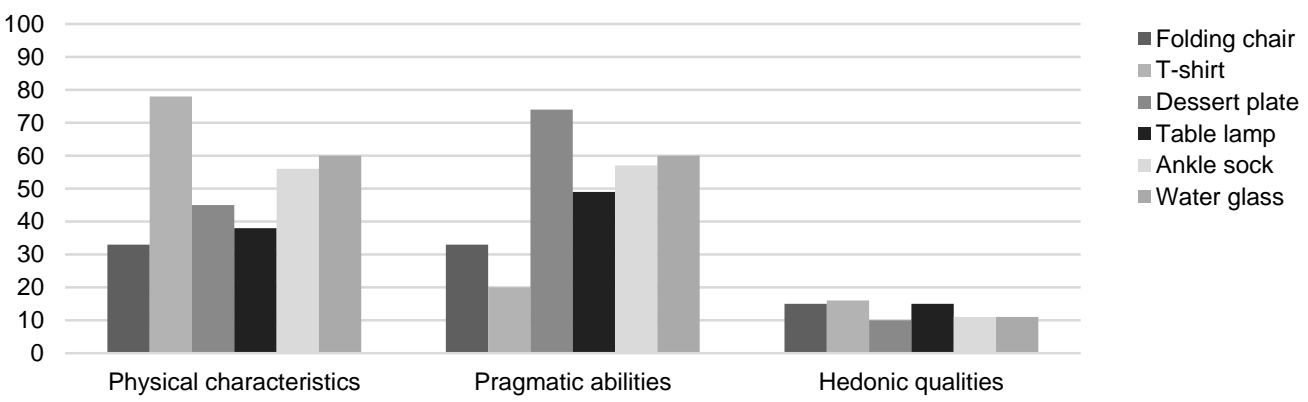

b

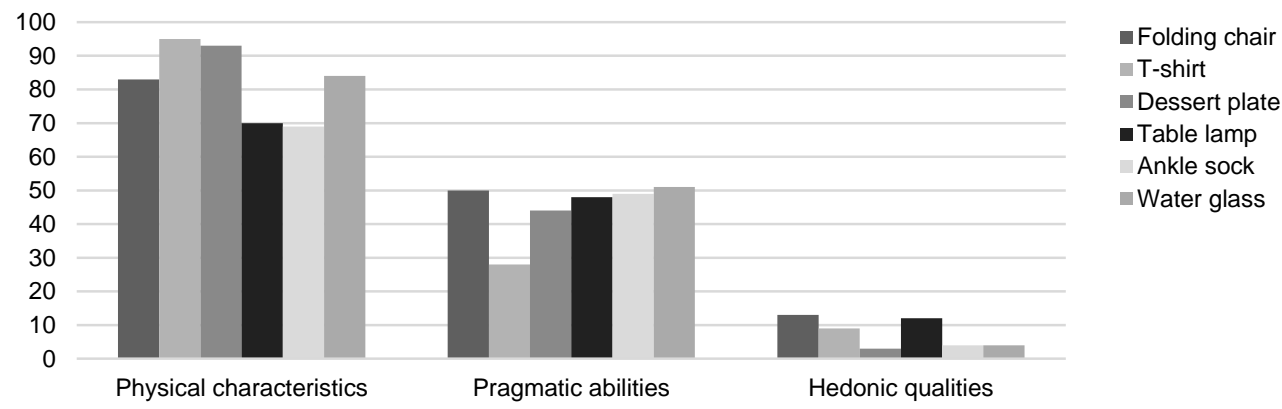

Figure 1. Frequencies of property types in word condition (a) and object condition (b)

A comparison of the average frequencies of responses per concept between the two conditions showed no complete agreement. There was a higher average frequency of responses in the object condition than in the word condition (Figure 2). With 136 responses in the word condition and 146 responses in the object condition, the average frequency of responses between the two conditions differed by $7 \%$. Moreover, there were slight differences in frequencies of responses between the two conditions in five out of all six concepts (Figure 3). A higher frequency of responses in the object condition than in the word condition was also observed for four out of all six concepts.

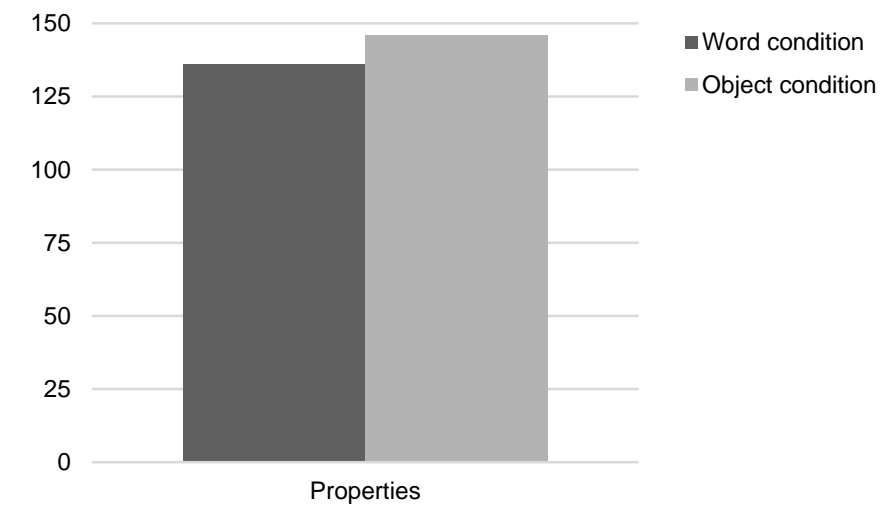

Figure 2. Frequencies of property responses in word condition and object condition 


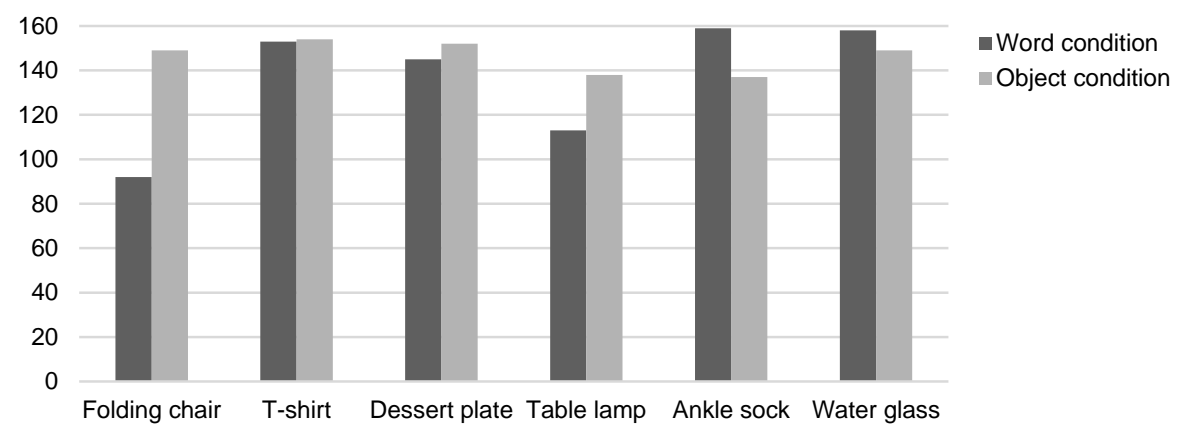

Figure 3. Frequencies of property responses in word condition and object condition for each concept

\section{DISCUSSION}

\subsection{Findings}

The present study set out to investigate the effects of experienced modality on perception of conceptual coherence. The studies showed that different representation modes of the same concept can elicit different perceptions of conceptual coherence for the concept.

The findings showed that the written word representation and the physical object representation generally elicited different but similar quantities of responses. This indicates similarity in levels of mental activity. When responding to concepts about their properties, a word and an object can be similarly stimulating for the mental activity. However, regarding property types, the written word representation elicited physical characteristic properties less often and pragmatic ability properties and hedonic qualities more often than the physical object representation. At the same time, regarding individual properties, the two representations elicited similar properties in different relative quantities, as did they elicit conflicting properties; especially properties observable in the physical object representation. These indicate difference in semantic processing. A word representing a concept is susceptible of many interpretations, as it provides little or no information about the concept that it represents. It is also is a more accurate representation of the general concept, as it can be used to label any of its instances. Contrarily, an object representing a concept is displaying its properties, so it provides immediate and direct information about itself. It is also an exact representation for a specific conceptual instance, and it cannot be used to illustrate all instances of its concept. This indication was also suggested in the pilot studies of the present study, where the participants considered the written word representation at a basic level and the physical object representation at a subordinate level. Basic level representations display a higher degree of concept inclusion than does subordinate level representations (Rosch et al., 1976), so participants indicated perception of the written word as a concept but of the physical object as a conceptual instance. When responding to concepts about their properties, a word that is multivalent and an object that is indicative can prompt different semantic processing, thus eliciting responses of different semantic content for two different representation modes.

The findings also showed that the two representation modes elicited different quantities of responses for five of the same concepts, the physical object representation elicited a higher quantity of responses for four of the same concepts, and the written word representation elicited more varied distribution of property types for all concepts. This indicates a possible influence of concepts on perception of conceptual coherence, which can be investigated further.

Generally, the findings of the present study indicate that a written word representation and a physical object representation for the same concept can call to mind similar quantities of properties with different semantic content, and that quantity and semantic content may be influenced by the concept. The indications of this finding are in line with the suggestions that perceptions of conceptual coherence for the same concept can differ (Norman, 2013), can depend on modality (Schifferstein and Hekkert, 2008), and may depend on concept (Kahlaoui et al., 2007). 


\subsection{Limitations}

Despite the attempts to consider factors that could impact the study and its findings, the study has several limitations. One limitation is the lack of prior research on the topic of perception of conceptual coherence in the design of physical products. The topic intrinsically refers to knowledge from other disciplines, from which knowledge was drawn to support the understanding of the research. Empirical studies can be conducted to build more pertinent knowledge for support.

Another limitation is the use of the method of feature listing to examine conceptual coherence. Studies in other disciplines have effectively used the same method to elicit syntheses of properties of concepts (e.g., McRae et al., 2005; Rosch et al., 1976), thus it was used to elicit perception of conceptual coherence in the present study. Different methods can be compared to find the most appropriately accurate way to examine conceptual coherence.

Yet another limitation is the sample that was drawn by convenience and therefore restricted by availability, which resulted in lacking representation. Individuals of the culture in which the concepts presented in the study are common were included to represent the population of interest. A more culturally consistent sample can be included to provide stronger indications of significant findings from the study.

Yet another limitation is the collected data of self-report responses that rely on cognition, which can cause discrepancy between the mental responses of interest and the actual responses provided. Participants were made aware that their responses were anonymised to lessen the pressure of representation and responsibility. Questions or tasks prompting the same response of interest can be provided in different ways to observe whether responses are in line with each other.

One almost inevitable limitation of the study is the nature of the study, that was a somewhat controlled study. While a highly controlled study better supports identification of actual relationships between concepts within the phenomenon studied, the high control may make the study reflect an oversimplified reality.

\subsection{Implications}

In the present study it was found that representation mode influences perception of conceptual coherence; not significantly in terms of mental activity, but considerably in terms of semantic processing. Since different modalities have different effects on perception of conceptual coherence, the use of them as representations of design must be considered comprehensively.

As a tool for designing products, representation of design elicits insights about the design to progress towards products for people. For the purpose of designing physical products that are understandable, designers should use design representations that are accurately indicative of the concept but otherwise multivalent in order to gain insight about the user's perception of conceptual coherence; that is, the synthesis of properties that they perceive as being coherent for the concept. Accurate indication implies the intention of the product, while multivalence evokes thoughts pertinent to the concept. The concept indication must not necessarily be a concept label, like chair or bottle; but it may be a utilitarian purpose, such as can be slept on or can fly, or may be an expressive purpose, such as minimalistic or royal. The more it is indicative of the just the concept and not any properties, the more appropriately it can be used to explore conceptual coherence.

With more awareness of the properties that are perceived to be coherent for a concept, the designer can design products that are not only understandable but also innovative. Properties can be evaluated on their centrality to a concept, which will inform about the degree to which they are mutable without altering the concept (Sloman et al., 1998). For the design of products for people, conceptually central properties can be strengthened for understandability to allow effortless use, while other properties can be changed for innovativeness to provide enjoyable oddity. Balance between familiarity and novelty is key (Schifferstein and Hekkert, 2008; Hekkert et al., 2003; Athavankar, 1989).

\section{CONCLUSION}

This paper presented a study that investigated conceptual coherence in the design of physical products. In a study investigating the effects of experienced modality on perception of conceptual coherence, participants responded with properties to the same concepts represented either as a written word or a physical object. It was found that representation mode does not significantly influence the mental 
activity towards perception of conceptual coherence, but does considerably influence the semantic processing towards perception of conceptual coherence.

The present study assessed the effects of written words and physical objects on perception of conceptual coherence. Apart from written words and physical objects, design can be represented by other modalities. Further research can study the effects of other modalities on perception of coherence in design. Similar to the present study, this can indicate how other representation modes influence perception and imply how they are to be used appropriately in the design of products.

Besides conceptual information, coherence can be perceived by sensory information or contextual information. Further research can explore sensory coherence and contextual coherence. Sensory coherence is especially interesting for the design of physical products, because physical products are innately multisensory, while contextual coherence is particularly important for the design of all products, because all products rarely, if ever, exist in isolation from context. This can inform designers about other types of coherence that can be considered in the design of products.

For a common foundation for the research on coherence in the context of design, a comprehensive conceptual framework for coherence should be established. Pertinent theories from other relevant disciplines can be drawn from to support existing theories about coherence in design. Coherence appears to be underexplored in design research. Thus, any broader and deeper understanding of the phenomenon will only support its presence in design research and practice.

\section{REFERENCES}

Alexander, C. (1973), Notes on The Synthesis of Form, Harvard University Press, Cambridge, England.

Anderson, J.R. (2015), Cognitive Psychology and Its Implications, 8th ed., Worth Publishers, New York, USA.

Athavankar, U.A. (1989), "Categorization... Natural Language and Design”, Design Issues, Vol. 5 No. 2, pp. 100-111. http://doi.org/10.2307/1511517.

Bowman, D. (2006), "Effective Product Platform Planning in the Front End", In: Simpson, T.W., Siddique, Z. and Jao, J.R. (Eds.), Product Platform and Product Family Design: Methods and Applications, Springer, New York, USA, pp. 19-26. http://doi.org/10.1007/0-387-29197-0_2.

Brereton, M. (2004), "Distributed Cognition in Engineering Design: Negotiating between Abstract and Material Representations", In: Goldschmidt, G. and Porter, W.L. (Eds.), Design Representation, 1st ed., SpringerVerlag London, pp. 83-103. http://doi.org/10.1007/978-1-85233-863-3_4.

Burnston, D. and Cohen, J. (2012), "Perception of Features and Perception of Objects", Croatian Journal of Philosophy, Vol. 12 No. 3, pp. 283-314.

Chitturi, R., Raghunathan, R. and Mahajan, V. (2007), "Form versus Function: How the Intensities of Specific Emotions Evoked in Functional versus Hedonic Trade-Offs Mediate Product Preferences", Journal of Marketing Research, Vol. 44 No. 4, pp. 702-714. http://doi.org/10.1509/jmkr.44.4.702.

Crilly, N., Maier, A. and Clarkson, P.J. (2008), "Representing Artefacts as Media: Modelling the Relationship Between Designer Intent and Consumer Experience", International Journal of Design, Vol. 2 No. 3, pp. $15-27$.

Dilkina, K. and Lambon Ralph, M.A. (2012), "Conceptual structure within and between modalities", Frontiers in Human Neuroscience, Vol. 6, pp. 1-26. http://doi.org/10.3389/fnhum.2012.00333.

Gershenson, J.K., Prasad, G.J. and Zhang, Y. (2003), "Product modularity: Definitions and benefits", Journal of Engineering Design, Vol. 14 No. 3, pp. 295-313. http://doi.org/10.1080/0954482031000091068.

Ginns, P. (2005), "Meta-analysis of the modality effect", Learning and Instruction, Vol. 15 No. 4, pp. 313-331. http://doi.org/10.1016/j.learninstruc.2005.07.001.

Goldschmidt, G. and Porter, W.L. (2004), Design Representation, 1st ed., Springer-Verlag London. https://doi.org/10.1007/b97667.

Hekkert, P., Snelders, D. and Van Wieringen, P.C.W. (2003), “"Most advanced, yet acceptable': Typicality and novelty as joint predictors of aesthetic preference in industrial design", British Journal of Psychology, Vol. 94 No. 1, pp. 111-124. http://doi.org/10.1348/000712603762842147.

Kahlaoui, K., Baccino, T., Joanette, Y. and Magnié, M.-N. (2007), "Pictures and Words: Priming and Category Effects in Object Processing", Current Psychology Letters, Vol. 3 No. 23, pp. 1-13.

Karjalainen, T.-M. (2003), "Strategic Brand Identity and Symbolic Design Cues", 6th Asian Design Conference, October 14-17, Science of Council of Japan, Tsukuba, Japan, pp. 1-13.

Kidd, M.W. and Thompson, G. (2000), "Engineering design change management", Integrated Manufacturing Systems, Vol. 11 No. 1, pp. 74-77. http://doi.org/10.1108/09576060010303686.

Kotler, P. and Armstrong, G. (2012), Principles of Marketing, 14th ed., Pearson Education, Inc., New Jersey, USA. 
McRae, K., Cree, G.S., Seidenberg, M.S. and McNorgan, C. (2005), "Semantic feature production norms for a large set of living and nonliving things", Behavior Research Methods, Vol. 37 No. 4, pp. 547-559. http://doi.org/10.3758/BF03192726.

Medin, D.L. (1989), “Concepts and Conceptual Structure”, American Psychologist, Vol. 44 No. 12, pp. 14691481. http://doi.org/10.1037/0003-066X.44.12.1469.

Murphy, G.L. (1991), "Parts in object concepts: Experiments with artificial categories", Memory \& Cognition, Vol. 19 No. 5, pp. 423-438. http://doi.org/10.3758/BF03199563.

Murphy, G.L. and Medin, D.L. (1985), "The Role of Theories in Conceptual Coherence”, Psychological Review, Vol. 92 No. 3, pp. 289-316. http://doi.org/10.1037/0033-295X.92.3.289.

Norman, D. (2013), The Design of Everyday Things, Revised and expanded edition, Basic Books, New York, USA.

Oxford Dictionary of English: Apple Inc. (2005/2018) Oxford Dictionary of English (British English)

Rosch, E., Mervis, C.B., Gray, W.D., Johnson, D.M. and Boyes-Braem, P. (1976), "Basic Objects in Natural Categories", Cognitive Psychology, Vol. 8, pp. 382-439. http://doi.org/10.1016/0010-0285(76)90013-X.

Schifferstein, H.N.J. and Desmet, P.M.A. (2008), "Tools Facilitating Multi-sensory Product Design", The Design Journal, Vol. 11 No. 2, pp. 137-158. http://doi.org/10.2752/175630608X329226.

Schifferstein, H.N.J. and Hekkert, P. (2008), Product Experience, 1st ed., Elsevier Science, San Diego, USA. https://doi.org/10.1016/B978-0-08-045089-6.X5001-1.

Sloman, S.A., Love, B.C. and Ahn, W.K. (1998), "Feature Centrality and Conceptual Coherence", Cognitive Science, Vol. 22 No. 2, pp. 189-228. http://doi.org/10.1207/s15516709cog2202_2.

Smith, E.E. and Medin, D.L. (1981), Categories and Concepts, 1st ed., Harvard University Press, Cambridge, England.

Smith, P.G. (2007), Flexible Product Development: Building Agility for Changing Markets, 1st ed., Jossey-Bass, San Fransisco, USA.

$\mathrm{Su}, \mathrm{H}$. and Hao, N. (2010), “The Principles for Modern Product Design”, in Chunqing, Y. (Ed.), International Conference on Information Technology and Scientific Management 2010, December 20-21, Scientific Research Publishing Inc., Tianjin, China, pp. 1-3.

Tanaka, J.W. and Taylor, M. (1991), "Object Categories and Expertise: Is the Basic Level in the Eye of the Beholder?”, Cognitive Psychology, Vol. 23 No. 3, pp. 457-482. http://doi.org/10.1016/00100285(91)90016-H.

Thomke, S. and Reinertsen, D. (1998), “Agile Product Development: Managing Development Flexibility in Uncertain Environments”, California Management Review, Vol. 41 No. 1, pp. 8-30. http://doi.org/10.2307/41165973.

Townsend, J.D., Montoya, M.M. and Calantone, R.J. (2011), "Form and Function: A Matter of Perspective", Journal of Product Innovation Management, Vol. 28 No. 3, pp. 374-377. http://doi.org/10.1111/j.15405885.2011.00804.X.

Ullah, I., Tang, D. and Yin, L. (2016), "Engineering product and process design changes: A literature overview”, 9th International Conference on Digital Enterprise Technology - DET 2016 - Intelligent Manufacturing in the Knowledge Economy Era, March 29-31, Nanjing, China, pp. 25-33. http://doi.org/10.1016/j.procir.2016.10.010.

Ulrich, K.T. and Eppinger, S.D. (2012), Product Design and Development, 5th ed., The McGraw-Hill, New York, USA.

Unsworth, S.J., Sears, C.R. and Pexman, P.M. (2005), "Cultural influences on categorization processes”, Journal of Cross-Cultural Psychology, Vol. 36 No. 6, pp. 662-688. http://doi.org/10.1177/0022022105280509.

Vosniadou, S. (2002), "Mental Models in Conceptual Development”, In: Magnani, L. and Nersessian, N.J. (Eds.), Model-Based Reasoning, 1st ed., Springer, New York, USA, pp. 353-368. http://doi.org/10.1007/978-1-4615-0605-8_20. 